\title{
Research on equipments and production line of hot stamping
}

\author{
Liang WANG, Bin ZHU and Yisheng ZHANG* \\ ${ }^{1}$ State Key Laboratory of Material Processing and Die \& Mould Technology, Huazhong University of \\ Science and Technology, Wuhan 430074, P.R. China \\ *zhangys@mail.hust.edu.cn
}

Keywords: Hot stamping; Automatic production line; Servo press; Multi-chamber furnaces; Fast transportation;

Abstract. Hot stamping is a reliable way for the production of high performance auto parts with light weight. Hot stamping process requires pre-blanking steel sheet to be transported and processed at elevated temperature, and the performance of hot stamping products is closely related to temperature. This paper presents 2 types of hot stamping lines independently developed, $2000 \mathrm{kN}$ test line and $6000 \mathrm{kN}$ production line. The $2000 \mathrm{kN}$ test line is composed of a chamber furnace for heating blanks, a $2000 \mathrm{kN}$ electric servo mechanical press for forming heated blanks and a joint robot for transporting. The production line has multi-chamber furnaces, high-speed multi-axis Cartesian robots and electric servo mechanical press. Hot stamping process is carried out by heating equipments, transporting equipments and forming equipments cooperatively. Mechanical link servo press (MLSP) carries out hot stamping process by fast downward, slowly mold closing and holding pressure at bottom. Robots cooperatively transport hot blanks as fast as possible for the sake of reducing exposure at air and temperature drop. Multi-chamber furnaces dispatch chambers into or out of production cycle to implement flexible production. The production line carries out continuous and efficient production at a steady beat.

\section{Introduction}

Hot stamping is a positive method for strength enhancement and weight reduction. Compared with traditional stamping process, hot stamping process is accompanied by dramatic change of heat, and it consumes much energy during heating. The life cycle of automobile is usually more than 10 years. When hot stamping technology is applied to automobile parts, weight reduction of automobile contributes to energy conservation in life cycle.
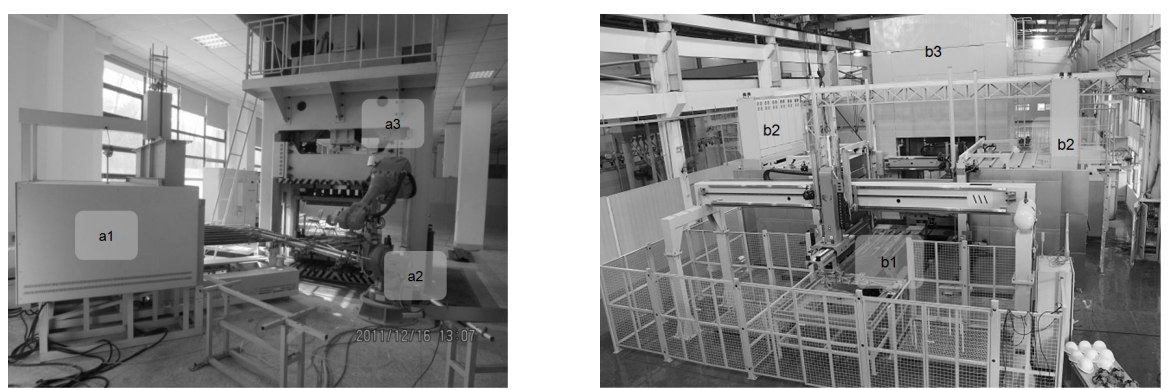

Fig. 1. The 2000kN Hot Stamping Test Line and SIGMA6000 Hot Stamping Line [1].

a1) box furnace, a2) multi-joint robot, a3) $2000 \mathrm{kN}$ electric servo mechanical press, b1) multi-axis Cartesian robots, b2) multi-chamber furnaces, b3) 6000kN electric servo mechanical press

This paper mentions 2 types of hot stamping line locally developed, hot stamping test line [2] and production line [3]. The test line consists of a box furnace for blank heating, a $2000 \mathrm{kN}$ electric servo press $[4,5]$ for stamping and quenching, a joint robot for blank transportation before and after heating up. The production line consists of 2 multi-chamber box furnace, 7 high-speed multi-axis Cartesian robots, a $6000 \mathrm{kN}$ electric servo press. These 2 lines are fully digital-controlled and flexible. The servo press has enough ability to adjust holding cycle, stamping speed and motion according to process demands. The box furnace offers anti-oxidation atmosphere and heats blanks to temperature above $950^{\circ} \mathrm{C}$. The joint robot in test line also coordinates operating order of the press and furnace. Cartesian 
robots in production line deliver blanks cooperatively. The local developed lines are energy-efficient and environment-friendly.

\section{Control Modeling of Hot Stamping Production Line}

Hot stamping line consists of different equipments with various controller and its automation involves integration and interaction of controllers, control flow and information flow. These limitations require that control model of hot stamping line has good compatibility with a wide range of adaptability.

The transportation process is obviously more complicated than heating or forming process. Robots should transport in designed time window and cooperate accurately with other robots and equipments. So transportation is the key of automation of this type of hot stamping line. Efficient cooperation of robots is the precondition for high efficient automation.

An improved RCS reference model [6] is presented for multi-unit cooperation in this paper, shown in Figure 1. The improved RCS model is a multi-layer and multi-resolution architecture consisting of compute nodes. At the lower layer, these elements generate goal-search-oriented reactive behavior, while at the higher layer, produce a goal-definition-oriented deliberate behavior. Through the grading process structure, interactions between the functional elements can produce behaviors like perception, cognition, imagination, reasoning, and so on. At the lower layer, the process area in time and space is small and the information processed is in a high resolution. But at the higher layer, the process area in time and space is wide and the information processed is in a low resolution. This approach enables that behaviors which need high accuracy and rapid response can be handled at a lower layer within a smaller space over a short period of time, while long-term planning and abstract concepts can be formed and realized at a higher layer within large spatiotemporal scope. This is the core of RCS of hierarchical intelligence.

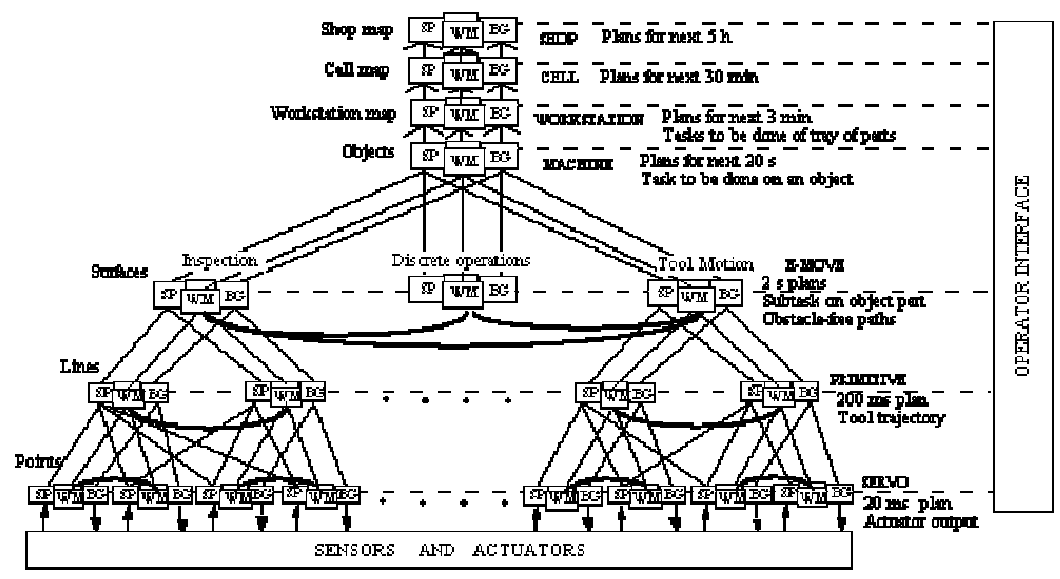

Fig. 2. An RCS reference model architecture for a machining center [7].

According to improved RCS reference model, control system of hot stamping line can be divided into 2 layers: command layer and task layer. The command layer includes several command nodes and is responsible for task planning, process control, map creation and analysis. The task layer contains multiple task execution nodes and complete special tasks. According to different designs of production line, the number and function of node can be adjusted at each layer, but the overall structure remains the same. Communication between nodes is via industrial-grade links.

\section{Equipments and Production Line of Hot Stamping}

SIGMA hot stamping line consists of 3 main parts: Production control system (PCS), manufacturing equipments (press, transporting robots, furnaces, gas, safety fence), Production supporting system (PSS). PCS is the center module of intelligent hot stamping line, coordinates a variety of manufacturing equipments and exchanges operating data with PSS. Field screw adjust manufacturing 
process and response to operating request through PCS. Production supervisors view and adjust manufacturing schedule by connecting to PSS.

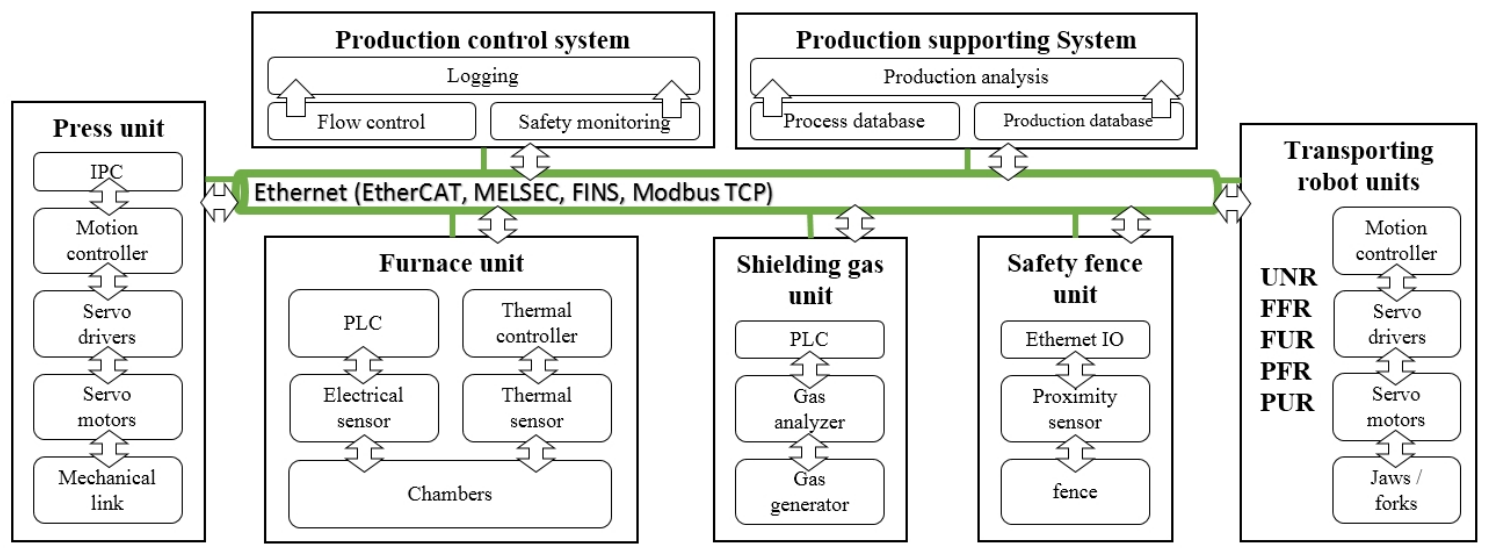

Fig. 3. System structure and workflow of hot stamping line

Hot stamping line uses Mechanical link servo press (MLSP) as forming device. In terms of performance, MLSP is capable of high-speed downward and upward, achieving more than 15 strokes per minute. MLSP uses servo motor as a power source, achieving more than $6000 \mathrm{kN}$ force output through multi-motor coordination, steady and stable output by multiple linkage structure. Controller of MLSP provides to the Production Control System (PCS) displacement, speed of slide and other status information, receives from PCS control commands and emergency instructions. In the process of stamping, hot blanking losses temperature while the slide of press is punching. Fast downward procedure of MLSP reduces temperature loss. When die closing, hot blank is easy to be fractured by drawing. So in this period, MLSP needs to be slowly to avoid fracture. At dwell stage, the better the surface contact of the tool to the part, the faster the cooling has been. In the hot stamping process the blank can thin up to $20 \%$ of its original thickness. It is possible that the output pressure of press could decrease because of blank thinning. Considering of this, the MLSP monitors to detect pressure drop and compensate pressure to guarantee proper contact between tool and material.

Furnace is adopted to heat boron-alloyed steel to target temperature and target micro-structure. This hot stamping line adopts multi-chamber box furnace. Working process of furnace is divided into 3 stages: heating stage, insulating stage and cooling stage. The furnace controls these 3 stages independently because heating stage and cooling stage takes several to more than 10 hours. In order to preventing devastating deformation of structure parts leaded to by thermal shock, temperature of furnace rises or falls smoothly during heating stage or cooling stage. In the insulating stage, a dynamic stable temperature are maintained. There are 14 chambers in 2 furnaces which are arranged in queues for availability management. Chambers has 4 status: available, blank heating, blank heating complete, blank heating timeout. There are 4 queues for 4 status. In these 4 queues, chambers follow FIFO principle and flow with the change of blank status. Chambers is not allowed to be sent into or taken out in blank-heating status. Chambers can be set to join or leave production cycle in the screen of PCS. The chambers are dispatched into or out of production cycle to implement flexible production.

This hot stamping line includes 5 transporting Cartesian robots, namely Press Feeding/Unloading Robot (PFR/PUR), Furnace Feeding/Unloading Robot (FFR/FUR), Unstacking Robots (UNR). UNR and FFR send cold blanks into chambers of the furnace, FUR and PFR take heated blanks out from furnace into dies on MLSP. FFR and FUR send blanks into and take them from chambers under collaboration. PFR and PUR also under collaboration send heated blanks into and remove parts from dies. 18 axis from 5 robots in total, are divided into 2 control stacks according their association. Stack 1 contains UNR and Stack 2 contains the other 4 robots. Robots in Stack 2 need to perform lots of multiple robots cooperations, which are easy to actualize by sharing status through local memory when participant robots are in the same control stack. The stacks of robots communicate as a unit with PCS. The stacks of robots response to status collection request and execute commands from PCS. 
Robots cooperatively transport hot blanks by parallel operation as fast as possible for the sake of reducing exposure at air and temperature drop.

Workflow of SIGMA hot stamping line is shown in Fig.3. Field crew entry PCS, set configuration parameters or load them from process database, deploy them to equipments. Parameters actually used will be recorded into the process database automatically.

The line starts after configuration. First, furnaces are heating up in a couple of hours to reach set temperature. Second, PCS dispatches unstacking robots and furnace feeding robots to deliver blanks into chambers of furnaces. Blanks heats up for $300 \sim 350$ seconds to $900 \sim 950{ }^{\circ} \mathrm{C}$ in furnaces. Third, PCS analyzes operating state of press and furnaces. If the press is available, PCS dispatches furnace unloading robot and press feeding robot to deliver heated blank from a chamber to die on the press. Once a piece of heated blank is taken out of the chamber, the chamber will be sent into with a piece of cold blank immediately.

PCS is always checking and recording operating and fault details of the line and these data is written into PSS. Fault data is for failure analyses in a short time scope, and for stability and reliability analyses in a longer time scope to predict and schedule equipments maintenance. Operating data is to analyze equipments performance and manufacturing management.

\section{Summary}

This paper presents a hot stamping test line and a production line locally developed. Both lines adopt electrical mechanical linkage servo press and low-heat-capacity box furnace. Blank or part transporting tasks are performed by a joint robot in the test line and 7 high-speed multi-axis Cartesian robots in the production line. The lines are high energy efficiency and environment friendly.

The control system of hot stamping production line is designed based on improved RCS reference model. The entire control architecture is reformed from traditional centralized control to distributed control, decoupled and layered. Mechanical link servo press (MLSP) carries out hot stamping process by fast downward, slowly mold closing and holding pressure at bottom. Robots cooperatively transport hot blanks as fast as possible for the sake of reducing exposure at air and temperature drop. Multi-chamber furnaces dispatch chambers into or out of production cycle to implement flexible production. The production line carries out continuous and efficient production at a steady beat.

\section{Acknowledgements}

This research work is financially supported by the National Natural Science Foundation of China (Grant No. 51275185).

\section{References}

[1] L. Wang, et al.: Production Control and Optimization of Hot Stamping Line. In: CHS2, Toronto, Canada. 2015.

[2] Wang Z.: Research on Total Control System of Flexible Hot Forming Production Lin. Thesis of the Master Degree of Engineering. 2013.

[3] L. Wang, et al.: High efficiency and low energy consumption electric servo press for hot stamping of high strength steel. In: CAMS 2014. Nov. 25-27, 2014.

[4] Mo J. H., et al.: The Properties and Operation of Large Mechanical Linkage Servo Press. In: China Metal Forming Equipment \& Manufacturing Technology, (2009), No. 5, pp 35-40.

[5] Osakada K., et al.: Mechanical servo press technology for metal forming. In: CIRP Annals Manufacturing Technology 60 (2011), No. 2, pp 651-672.

[6] http://www.nist.gov/el/isd/rcs_ref_model.cfm

[7] NIST.: An Intelligent Systems Architecture for Manufacturing (ISAM): A Reference Model Architecture for Intelligent Manufacturing Systems. In:

http://www.isd.mel.nist.gov/projects/rcs/isam/ISAM_web.htm. 